\title{
Profils épidémio-cliniques des enfants atteints de COVID-19 à Lomé.
}

\section{Epidemiological and clinical profiles of children with COVID-19 in Lomé.}

Bawe LD ${ }^{1,2}$, Kotosso $A^{1,2,3}$, Abaltou $B^{1,2,3}$, Assenouwe $A^{2,5}$, Aziagbe $\mathrm{KA}^{2,4}$, Tchamdja $\mathrm{G}^{2,5}$, Adougba $\mathrm{AC}^{2}$, Mossi $\mathrm{KE}^{2,5}$,Biaou $\mathrm{DM}^{2,}{ }^{4}$,Blatome $\mathrm{LO}^{2,5}$, Maman $\mathrm{S}^{2,5}$,Bekemsi $\mathrm{M}^{2,5}$,Assih $\mathrm{D}^{2,5}$,Nebona L ${ }^{2,5}$, Afoda $\mathrm{KM}^{2}$, Kanaza $\mathrm{GE}^{2}$, Akpodo $\mathrm{AD}^{2}$, Hoehanou $\mathrm{AE}^{2}$, Patassi $\mathrm{AA}^{1}$,Adjoh $\mathrm{KS}^{2,4}$, Wateba $\mathrm{MI}^{1,2}$

1 : Service des maladies infectieuses, CHU Sylvanus Olympio (Lomé, Togo)

2 : Centre hospitalier régional Lomé commune

3 : Centre hospitalier des Armées de Lomé

4 : Service de pneumologie, CHU Sylvanus Olympio (Lomé, Togo)

5 : Clinique médico-chirurgicale, CHU Sylvanus Olympio (Lomé, Togo)

Auteur correspondant : LD BAWE ; Email : alainbawe@yahoo.fr ;

\section{Résumé}

Objectif : Décrire les caractéristiques épidémiologiques, cliniques et thérapeutiques chez les enfants atteints de COVID-19 à Lomé. Patients et Méthodes : Il s'agissait d'une étude rétrospective allant du 6 mars au 13 juin 2020 soit 3 mois après la notification du premier cas au Togo ; menée au CHR Lomé-commune, centre de prise en charge de la COVID-19 à Lomé. Etaient inclus tous les enfants dont l'âge était inférieur ou égal à 17 ans avec une RT-PCR positive sur les prélèvements nasopharyngés. Résultats : Dix-huit enfants sur 274 cas confirmés ont été notifiés soit un taux de 6,6\%. On dénombrait 11 garçons et 7 filles. Leur âge moyen était de 9,1 ans avec des extrêmes de 28 jours à 17 ans. Les présentations cliniques étaient réparties comme suit: 15 asymptomatiques ; 2 formes légères et une forme critique. Les signes légers étaient marqués par un écoulement nasal ou un rhume (1 cas), une toux sèche (1 cas), une irritation de la gorge (1 cas) et une asthénie respectivement (1 cas).-Le protocole Chloroquine/Azithromicine a été administré chez 15 enfants.-Le décès a été enregistré chez la forme critique.-La durée moyenne d'hospitalisation était de 14,8 jours [6 - 25 jours].Conclusion: L'infection au nouveau coronavirus chez les enfants est le plus souvent asymptomatique. Mots clés : COVID-19, enfant, centre hospitalier régional Lomé commune, Togo.

\begin{abstract}
Objective: Description of the epidemiological, clinical and therapeutic characteristics in children with COVID19 in Lomé. Design: This was a retrospective study from March 6 to June 13, 2020, 3 months after the first case report in Togo.This study was conducted at the CHR Lomé-commune, a COVID-19 management center in Lomé and covered all the children up to 17 years of age with positive RT-PCR on nasopharyngeal swabs. Results: Eighteen children out of 274 confirmed cases were reported, that is, a rate of $6.6 \%$. This included 11 boys and 7 girls. Their average age was 9.1 years with extremes of 28 days to 17 years. The clinical presentations were distributed as follows: 15 asymptomatic; 2 mild forms and one critical form. The mild signs were marked by nasal discharge or cold ( 1 case), dry cough ( 1 case), throat irritation ( 1 case) and asthenia ( 1 case).- 15 children were treated with the Chloroquine/Azithromicin protocol treatment.-The death was recorded in the critical form. The average length of hospitalization was 14.8 days [6 - 25 days].Conclusion: The infection with the new coronavirus in children is most often asymptomatic. Key words: COVID-19, child, Centre Hospitalier Regional Lomé commune, Togo.
\end{abstract}

\section{Introduction}

Parmi les pandémies les plus connues, on retrouve la peste noire, ayant sévi au Moyen Âge, la grippe espagnole de 1918, le virus d'immunodéficience humaine (VIH) qui perdure depuis les années 1980, et la grippe A (H1N1) de 2009 [1]. La pandémie de la maladie à coronavirus (COVID-19) causée par le coronavirus du syndrome respiratoire aigu sévère 2 (SRASCoV-2) est toutefois sans précédent pour ces 100 dernières années en termes d'impacts sur l'activité humaine [1].Les personnes considérées les plus à risque de décéder à la suite de complications reliées à la COVID-19 sont les personnes âgées de plus de 65 ans, ainsi que les personnes atteintes d'une condition de santé sous-jacente, comme l'hypertension, le diabète, les maladies cardiovasculaires, les maladies respiratoires chroniques et le cancer [2]. Les personnes immunodéprimées sont aussi plus à risque de maladie sévère [2].L'information sur la COVID-19 demeure limitée chez les enfants et les données disponibles représentent un faible pourcentage des cas, soit moins de $1 \%$ [3]. La gravité et le taux de mortalité de cette maladie sont moindres dans cette tranche d'âge que chez les adultes $[4,5]$. Le Togo a enregistré son premier cas le 6 mars 2020, un sujet adulte. Les enfants représentent une faible proportion parmi les cas confirmés. Nous nous 
sommes intéressés aux enfants chez lesquels l'infection à SARS-Cov-2 symptomatique semble peu fréquente pour décrire les caractéristiques épidémiologiques, cliniques et thérapeutiques ; ainsi que les difficultés éventuellement rencontrées dans leur prise en charge au Centre Hospitalier Régional (CHR) Lomé-commune, centre de prise en charge de Covid-19 à Lomé au Togo.

\section{Patients et Méthode}

\section{Type d'étude et cadre d'étude}

Il s'agissait d'une étude rétrospective de 3 mois après la notification du premier cas au Togo, allant du 6 mars au 13 juin 2020 au CHR Lomécommune, centre de prise en charge de la COVID19 à Lomé. Le CHR Lomé-commune est l'hôpital du district sanitaire 2 sur les 5 districts sanitaires que compte la région sanitaire Lomé-commune. Le début de l'épidémie était confronté à un certain moment au problème de capacité d'accueil sur le site initial de prise en charge pour contenir tous les cas confirmés. C'est ainsi que le CHR Lomécommune, un hôpital construit en 2010 dans le cadre de la coopération sino-togolaise était la structure sanitaire qui pouvait valablement servir de centre de prise en charge.

\section{Population d'étude}

Elle concernait tous les enfants confirmés après la positivité de la RT-PCR sur les prélèvements nasopharyngés.

Etait considéré comme enfant dans notre série, tout patient dont l'âge était inférieur ou égal à 17 ans.

\section{Définitions opérationnelles}

Présentations cliniques [6]

La présentation de la maladie peut varier de l'absence de symptômes (patients asymptomatiques), à la pneumonie sévère et la mort. Les patients symptomatiques sont classés en légers ou modérés, sévères et critiques. Ainsi nous avons distingués 4 présentations cliniques :

-asymptomatique ;

-symptomatique léger ou modéré ;

-symptomatique sévère ;

-symptomatique critique.

Prise en charge [6]

La prise en charge a été faite selon le Protocole de prise en charge thérapeutique des cas de Covid-19 confirmés au Togo.
Le Togo a adopté une position réservée sur le traitement à base de l'Hydroxychloroquine/Chloroquine associée à l'Azithromicine. Chez l'enfant, ces médicaments sont administrés comme suit :

-Chloroquine ou Hydroxychloroquine : $10 \mathrm{mg} / \mathrm{kg}$ par jour répartis en trois prises quotidiennes pendant 10 jours associée à :

-Azithromycine : $20 \mathrm{mg} / \mathrm{kg}$ le premier jour puis 10 $\mathrm{mg} / \mathrm{kg}$ par jour du deuxième au cinquième jour. $\bullet$

\section{complications}

Traitement symptomatique et des

- Paracétamol en cas fièvre ;

- Réhydratation orale ;

- Vitamine C ;

- Amoxicilline et Acide Clavulanique ou Ceftriaxone si surinfection pulmonaire ;

- Anti histaminique (desloratadine, loratadine, méquitazine) si rhume ;

- Oxygénothérapie en cas de dyspnée avec fréquence respiratoire supérieure à 30 cycles par minutes et ou une saturation pulsée en oxygène inférieure à $92 \%$ (le débit doit être réglé pour avoir une saturation $\geq 92 \%$ ).

Les lunettes pour les débits entre 1 et 5 litres par minute ; par minute ;

Les masques pour les débits de 6 à 10 litres

Masque à haute concentration pour des débits supérieurs 10 litres par minute.

- Assistance ventilatoire à envisager si persistance de détresse respiratoire avec une saturation inférieure à 90 \% malgré l'oxygénothérapie.

Traitement des pathologies associées

Ces pathologies peuvent être préexistantes ou découvertes au cours de l'examen du malade.

\section{Contrôle RT-PCR}

La prise en charge des patients COVID-19 confirmés suite à un test RT-PCR confirmé se fait dans des centres clairement identifiés par le gouvernement quel que soit la symptomatologie (symptomatique ou asymptomatique). Après admission dans un centre de prise en charge, la séquence des contrôles RT-PCR se fait comme suit :

-Le premier contrôle prévu initialement au cinquième jour d'hospitalisation (J5) a été ramené au septième jour d'hospitalisation (J7) ;

-Si le premier contrôle du patient reste positif, le protocole avait prévu initialement un second test contrôle 5 jours après le premier test mais qui également a été ramené à 7 jours après le premier contrôle ;

-Si premier test contrôle est négatif, un autre est réalisé 3 jours après (tout test négatif pour la première fois est confirmé dans les 72 heures).

Critères de guérison [6]

Deux tests contrôles consécutifs négatifs de la charge virale par PCR à 3 jours d'intervalle déclarent le patient guéri.

En cas de premier test de contrôle négatif, le patient est maintenu en hospitalisation avec les mêmes mesures de barrière standard jusqu'au deuxième test négatif.

\section{Résultats}

Données épidémiologiques

Du 06 mars au 13 juin 2020, la situation épidémiologique de la pandémie de COVID-19 au 
Togo avait enregistré 525 cas confirmés dont 274 cas confirmés hospitalisés au CHR Lomé-commune ou confinés dans un hôtel de la capitale. Parmi ces cas confirmés, nous avons dénombré 18 enfants soit un taux de 6,6\%; dont 11 garçons et 7 filles. Leur âge moyen était de 9,1 ans avec des extrêmes de 28 jours à 17 ans.

La notion de contact avec un cas confirmé a été retrouvée chez 13 enfants; 6 enfants étaient contacts de leurs pères dont un décès de COVID-19 d'un père de famille. Parmi les 6 enfants nous avons enregistré 2 cas de recontamination après la guérison; cette fois-ci contacts de leur mère confirmée COVID-19. Les autres enfants étaient des contacts d'un cohabitant déclaré positif au COVID-19. La notion de contact était inconnue dans 4 enfants et une notion de voyage chez un enfant.

\section{Présentations cliniques}

Les présentations cliniques à l'admission des enfants étaient réparties comme suit: 15 asymptomatiques; 2 formes légères et une forme critique.

Dans la forme légère, les signes cliniques étaient marqués par un écoulement nasal ou un rhume (1 cas); une toux sèche (1 cas); une irritation de la gorge ( 1 cas); et une asthénie (1 cas). La RT-PCR était positive chez un nouveau-né de 28 jours de sexe féminin avec un syndrome polymalformatif (méningo-encéphalocèle + dysmorphie faciale) avec les signes suivants: difficultés respiratoires avec une polypnée à prédominance inspiratoire, un tirage intercostal, un bruit de cornage (stridor laryngé) avec un score de Silverman évalué à 6/10.

\section{Aspects thérapeutiques}

Le protocole Chloroquine/Azithromicine a été administré chez 15 enfants. Pour les 3 enfants restants, le phytomédicament malgache COVID ORGANICS en essai a été administré chez un enfant tandis que les deux autres n'avaient rien reçu comme traitement. Un traitement symptomatique a été fait dans tous les cas au besoin. En plus du protocole Chloroquine/Azithromicine, le nouveauné a bénéficié des mesures de réanimation à savoir : -Intubation oro-trachéale en séquence rapide + branchement au respirateur ;

-Sédation à base de Diazépam $(0,6 \mathrm{mg} / \mathrm{kg} /$ heure $)+$ Fentanyl (0,3y/kg/heure) ;

-Enoxaparine 300 UI dans $100 \mathrm{cc}$ de sérum physiologique ;

-Antibiothérapie à base de Ceftriaxone $250 \mathrm{mg}$ en intra-veineuse directe ;

-Paracétamol injectable en cas de fièvre ;

-Apport hydro-électrolytique 19,6 ml/heure à la séringue électrique ;

-Apport calorique à base du lait maternel 50 cc/2heures.

Aspects évolutifs
Douze cas de guérison ont été notifiés à l'issu d'une durée moyenne d'hospitalisation de 13,1 jours [7 24 jours]. Le décès est arrivé chez le nouveau-né après 48 heures d'hospitalisation. Cinq cas étaient encore actifs (dont un enfant avec un premier test contrôle PCR négatif) après une durée moyenne d'hospitalisation de 14,8 jours [ 6 - 25 jours].

Chez les guéris, la négativation de la PCR au premier test contrôle a été obtenu au $5^{\mathrm{e}}$ jour d'hospitalisation chez 8 enfants tandis que 2 enfants l'avaient obtenus après 7 jours d'hospitalisation.

\section{Discussion}

La COVID-19 est moins fréquente et moins grave chez l'enfant que chez l'adulte. Selon des données chinoises, les enfants représentent $1 \%$ des cas d'infections par le SRAS-CoV-2 quel que soit l'âge [7]. Depuis le déclenchement de la nouvelle infection à coronavirus 2019 (2019-nCoV) dans la ville de Wuhan en Chine, et au 30 janvier 2020, un total de 9692 des cas ont été confirmés dans 31 provinces ou villes de Chine. Parmi les cas confirmés, un total de 28 enfants âgés de 1 mois à 17 ans a été signalé en Chine [5]. Le rapport de la situation épidémiologique en Chine publié par l'Organisation Mondiale de la Santé le 28 février 2020, rapporte que les cas pédiatriques représentaient 2,4\% des 55924 cas confirmés [8]. En France, les données de Santé publique France du 21 avril 2020 confirment que les enfants sont peu représentés $(2 \%)$ dans la population de patients consultant aux urgences pour suspicion de COVID19 [9]. Entre le 16 janvier et le 8 février 2020, une série chinoise a porté sur 2143 enfants diagnostiqués d'âge médian 7 ans (2-13 ans), majoritairement de sexe masculin $(56,6 \%)$ [10]. Les données actuelles ont révélé que l'âge d'apparition de la maladie variait de 1,5 mois à 17 ans, et la plupart des enfants avaient eu des contacts avec des cas confirmés ou étaient des cas issus des clusters familiaux [11]. Dans une large étude rétrospective portant sur 171 patients âgés de moins de 16 ans, la contamination était essentiellement d'origine intrafamiliale (90 \%) [12]. Ainsi, la proportion des enfants infectés est variable d'un pays à l'autre en tenant compte du nombre total de cas confirmés ; mais le constat général est que les enfants sont peu touchés. Au plan clinique, les formes asymptomatiques semblent plus fréquentes chez l'enfant; estimées à environ $30 \%$ des cas. L'impact épidémiologique de ces formes asymptomatiques n'est pas encore clair, mais plusieurs cas de contamination durant la période d'incubation ou à partir de patients asymptomatiques ont été rapportés [13]. La plupart des enfants infectés présentent de légères manifestations cliniques comme la fièvre, la toux sèche et la fatigue; peu ont des manifestations des voies respiratoires supérieures incluant une congestion nasale et un écoulement du nez. Certains 
enfants présentent des symptômes gastrointestinaux notamment une gêne abdominale, des nausées, des vomissements, des douleurs abdominales et une diarrhée. Quelques fois, ils n'ont ni fièvre ni symptômes de pneumonie [5]. Les formes sévères ont été décrites chez des patients présentant des comorbidités et chez lesquels une hospitalisation en soins intensifs a été nécessaire [14]. Dans notre série, il s'agissait d'un nouveauné de 28 jours polymalformé dont les cas a été critique et fatal. Dans une série en Chine, aucun cas de contamination d'un nouveau-né issu d'une mère infectée n'a été rapporté et aucun cas de nouveauné n'a été également signalé [5]. Pour le cas du nouveau-né de notre série qui a présenté la forme critique, elle a été détectée positive le 17 avril 2020 soit 28 jours après sa naissance devant des manifestations respiratoires qu'elle a présenté. L'interrogatoire révèle que l'enfant a fréquenté plusieurs formations sanitaires entre la naissance et la positivité de son test RT-PCR dans le cadre de ses malformations; de plus la mère étant une commerçante a cessé toute activité depuis son accouchement. L'hypothèse d'une contamination lors des consultations est fortement évoquée. La mère n'a été détectée positive que par la suite. Le doute sur l'origine réelle de la contamination de l'enfant demeure toujours puisque la mère étant asymptomatique n'était pas suspectée. Il se peut également que cette dernière était contaminée et bien qu'asymptomatique ait contaminée son enfant. Mais il est important de dire que la symptomatologie présentée par le nouveau-né ne peut pas être imputable à la positivé de la RT-PCR, surtout que ce n'est pas soutenue par la littérature. Les enfants les plus à risque de forme grave sont probablement, comme chez l'adulte, ceux ayant une pathologie chronique: pulmonaire (insuffisance respiratoire chronique, bronchopathie, en particulier mucoviscidose, dyskinésie ciliaire primitive, dilatation des bronches, dysplasie bronchopulmonaire, asthme sévère, hypertension artérielle pulmonaire); insuffisance corticotrope ou surrénalienne; immunosuppression, en particulier leucémies aiguës lymphoblastiques; maladies inflammatoires auto-immunes ou autoinflammatoires non contrôlées; traitements immunosuppresseurs ; insuffisance cardiaque, cardiopathies cyanogènes; certaines maladies héréditaires du métabolisme [9]. Les cas pédiatriques sévères présentent une dyspnée évidente qui peut rapidement évoluer vers un syndrome de détresse respiratoire aiguë, choc septique, acidose métabolique réfractaire, trouble de la coagulation et défaillance multiviscérale. Ceux qui évoluent vers les formes critiques peuvent avoir une fièvre modérée ou sans fièvre apparente [15]. Le traitement de la COVID-19 est comme chez l'adulte, uniquement symptomatique, en l'absence de traitement spécifique d'efficacité démontrée [9]. Bien que certains antiviraux soient en cours d'essai clinique chez l'adulte, sans preuves claires de sécurité et d'efficacité, ils ne sont pas recommandés chez les enfants [15]. Ailleurs certains antiviraux comme lopinavir/ritonavir, ribavirine ou phosphate de chloroquine ne sont pas recommandés chez les enfants [15]. Dans notre contexte africain, la chloroquine est une molécule bien connue il y a plusieurs années utilisée jadis dans le traitement du paludisme mais abandonnée depuis longtemps à cause des résistances décrites sur le Plasmodium. La difficulté que nous avons eue chez certains enfants était l'absence des formes pédiatriques de Chloroquine chez certains enfants surtout le cas du nouveau-né. Les comprimés étaient divisés pour avoir la posologie approximative, puis écrasés et mélangés avec de l'eau pour l'administration au nouveau-né. La létalité obtenue concorde avec les données de la littérature [12]. Globalement, on admet donc que toutes les données sont rassurantes avec, chez l'enfant par rapport à l'adulte, 10000 fois moins de décès, 1000 fois moins de formes graves nécessitant une réanimation, 100 fois moins d'hospitalisations et 10 fois moins de sujets atteints [9].

\section{Conclusion}

Notre série confirme les données de la littérature concernant l'infection au nouveau coronavirus chez les enfants. Les cas asymptomatiques et les manifestations cliniques légères sont prédominants dans toutes les séries décrites ailleurs.

Les cas sévères ou critiques sont rencontrés chez les enfants de moins d'un an surtout avec des comorbidités comme décrit dans notre série.

Conflit d'intérêt : aucun

\section{Références}

1. Lapierre A, Fontaine G, Tremblay PL, MaheuCadotte MA, Desjardins M. La maladie à coronavirus (COVID-19) : portrait des connaissances actuelles. Soins d'Urgence, 2020;1 (1):13-8.

2. Institut National d'Excellence en Santé et en Services Sociaux (INESSS). COVID-19 et personnes immunodéprimées. Québec, Qc: INESSS;2020.

3. Wu Z, McGoogan JM. Characteristics of and important lessons from the coronavirus disease 2019 (COVID-19) outbreak in China: Summary of a report of 72314 cases from the chinese center for disease control and prevention. JAMA. 2020;323(13):1239-42.

4. Le Saux N. L'épidémiologie à jour sur la COVID-19 (causée par le virus SARS-CoV-2) chez les enfants et les conseils s'y rapportant : mars 2020. Société Canadienne de Pédiatrie, comité des maladies infectieuses et d'immunisation; 2020. 
5. Shen K, Yang Y, Wang T, Zhao D, Jiang Y, Jin $\mathrm{R}$, et al. Diagnosis, treatment, and prevention of 2019 novel coronavirus infection in children: experts' consensus statement. World J Pediatr. 2020 16(3):223-231. doi: 10.1007/s12519-02000343-7.

6. Conseil scientifique pour la gestion de la pandémie COVID-19 au Togo. Protocole de prise en charge thérapeutique des cas de Covid-19 confirmés au Togo. République Togolaise ; Version 001 du 17 Avril 2020, 14p.

7. Wu Z, McGoogan JM. Characteristics of and Important Lessons From the Coronavirus Disease 2019 (COVID-19) Outbreak in China: Summary of a Report of 72314 Cases From the Chinese Center for Disease Control and Prevention. JAMA. 2020; 323(13):1239-42.

8. World Health Organization. Report of the WHOChina Joint Mission on Coronavirus Disease 2019 (COVID-19). World Health Organization;2020, 40p.

9. https://www.santepubliquefrance.fr

10. Dong Y, Mo X, Hu Y, et al. Epidemiology of COVID-19 Among Children in China. Pediatrics. 2020;145(6):e20200702.
11. The Society of Pediatrics of Hubei Medical Association, The Society of Pediatrics of Wuhan Medical Association, Hubei Pediatric Medical Quality Control Center. Suggestions on the diagnosis and treatment of novel coronavirus infection in children in Hubei province (trial version 1). CJCP. 2020;22:96-9 (in Chinese).

12. Lu X, Zhang L, Du H, Zhang J, Li YY, Qu J, et al. SARS-CoV-2 Infection in children. $\mathrm{N}$ Engl J Med 2020; 382:1663-5.

13. Bai Y, Yao L, Wei T, Tian F, Jin D-Y, Chen L, et al. Presumed asymptomatic carrier transmission of COVID-19. JAMA. 2020; 323(14) : 1406-1407.

14. Plaçais L, Richier Q. COVID-19 : caractéristiques cliniques, biologiques et radiologiques chez l'adulte, la femme enceinte et l'enfant. Une mise au point au cœur de la pandémie. La Revue de médecine interne. 2020;41(5):308-18. 15. Shen KL, Yang YH, Jiang RM, et al. Updated diagnosis, treatment and prevention of COVID-19 in children: experts' consensus statement (condensed version of the second edition) [published online ahead of print, 2020 Apr 24]. World J Pediatr. 2020;1-8. 